\title{
Secuelas de mastectomía subcutánea profiláctica: caso clínico
}

\section{Prophilactic subcutaneous mastectomy sequeal: case report}

\author{
Gutiérrez Gómez, C.*, Rivas León, B.**, Cárdenas Mejía, A.***
}

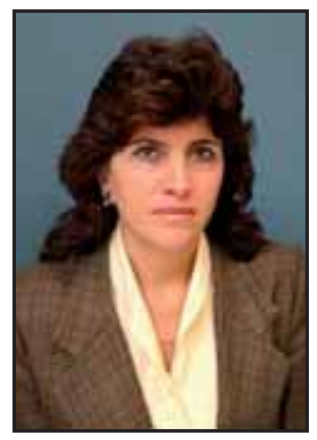

Gutiérrez Gómez, C.

\section{Resumen}

La mastectomía subcutánea profiláctica es un procedimiento controvertido, sin embargo, es un hecho que disminuye la incidencia de cáncer mamario hasta en un $100 \%$ en pacientes con alto riesgo de padecerlo. Presentamos el caso de una paciente de 26 años de edad a quien se le realizó dicho procedimiento sin una indicación precisa y que presentó complicaciones (dehiscencia de heridas, infección y exposición de ambas prótesis mamarias) que requirieron varios procedimientos reconstructivos, con un seguimiento de 8 años.

La mastectomía subcutánea profiláctica es un procedimiento irreversible con implicaciones importantes para las pacientes, por lo que consideramos que debe estar plenamente justificada por estrictos criterios de prevención de cáncer mamario en pacientes con alto riesgo.

\begin{tabular}{rl|} 
Palabras clave & Mastectomía profiláctica. Carcinoma \\
& mamario. Secuelas. \\
Código numérico & $5213-5214$
\end{tabular}

\begin{tabular}{|ll|}
\hline Key words & $\begin{array}{l}\text { Prophylactic mastectomy. Breast cancer. } \\
\text { Sequeal. }\end{array}$ \\
Numeral Gode & $5213-5214$ \\
\hline
\end{tabular}

Prophylactic subcutaneous mastectomy is controversial, in fact this procedure has proved its efficacy in decreasing mammary carcinoma in high risk patients up to 100 per cent. We report a case of 26 years old patient, in whom was performed a prophylactic bilateral subcutaneous mastectomy without a clear indication, and who presented several complications as suture dehiscence, infection and mammary implants exposition requiring several reconstructive procedures, with a follow up of 8 years.

Prophylactic subcutaneous mastectomy is an irreversible procedure with important implications for the patient; we consider that it should be limited to patients with precise indications and at high risk for mammary carcinoma.

\footnotetext{
* Cirujana Plástica. Profesora Adjunta curso postgrado de Cirugía Plástica y Reconstructiva, Universidad Nacional Autónoma de México. Hospital General "Dr. Manuel Gea González", Ciudad de México. México.

* Cirujano Plástico. Adscrito Instituto Nacional Cancerología Ciudad de México. México.

* Cirujano Plástico. Adscrito al Servicio Cirugía Plástica Hospital General “Dr. Manuel Gea González", Ciudad de México. México.
} 

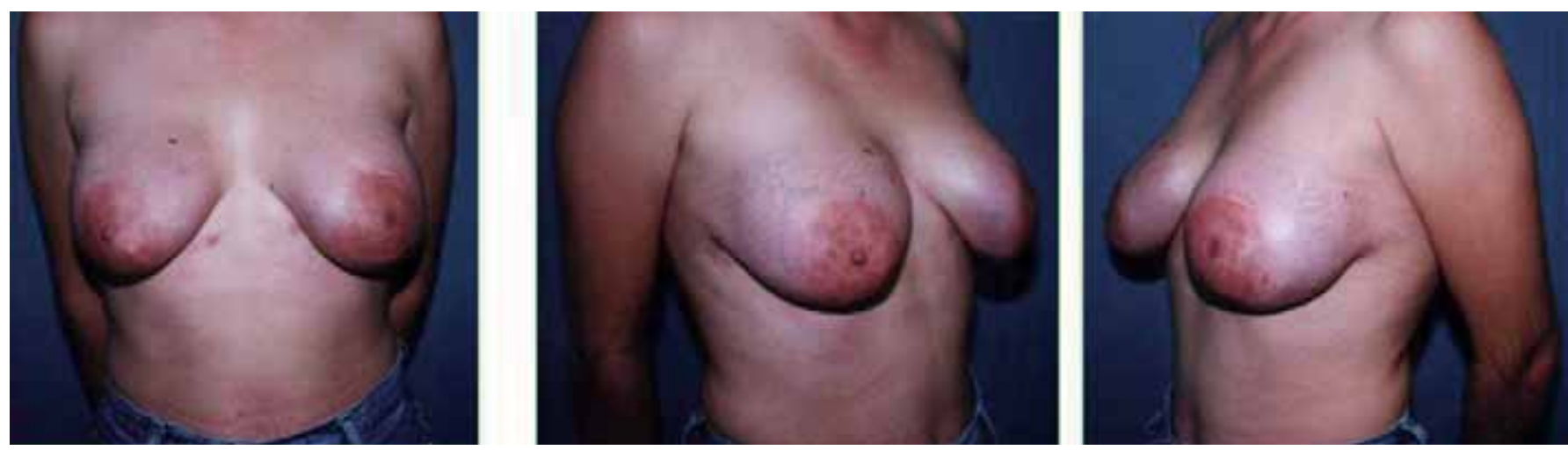

Fig. 1. Vistas frontal y de tres cuartos de la paciente, 2 meses después de mastectomía subcutánea bilateral profiláctica y colocación de prótesis redondas de solución salina de $400 \mathrm{cc}$.

\section{Introducción}

La mastectomía profiláctica se encuentra plenamente justificada para pacientes con riesgo elevado de cáncer mamario; los criterios para su indicación incluyen:

I.- Una o más familiares de primer grado (madre, hija, hermana) con antecedente de cáncer de mama durante la premenopausia. 2.-Dos o más familiares de primer grado con antecedentes de cáncer de mama en etapa postmenopáusica. 3.-Antecedentes de enfermedad macroquística, definida por la presencia de 5 o más quistes que han requerido aspiración. 4.-Antecedente de carcinoma lobular in situ demostrado por biopsia. 5.-Hiperplasia ductal atípica. 6.-Hiperplasia lobular atípica (1). Actualmente, las pacientes que presentan mutaciones en los genes BRCA1 o BRCA2 también tienen indicación para mastectomía subcutánea, con lo que reducen hasta un $100 \%$ en riesgo de padecer un cáncer mamario (2).

En los inicios de la mastectomía profiláctica, se desconocía su efectividad y se juzgaba como una medida extrema, drástica y desesperada $(3,4)$. Sin embargo, actualmente, se presenta una reducción del cáncer de mama en pacientes mastectomizadas de entre un 89,5 y un $100 \%$ (5-7).

La enfermedad macroquística es una alteración mamaria benigna caracterizada por la presencia de quistes con contenido líquido que se originan en la porción terminal de los conductos lobulares terminales de la mama, con una asociación de 2 a 4 veces mayor incidencia de cáncer en las pacientes afectadas (8-11).

El incremento en los casos de cáncer mamario, nos lleva en ocasiones a exagerar en las indicaciones de la mastectomía profiláctica; Chun et al refiren incluso (1) no haber encontrado un incremento estadísticamente significativo de carcinoma en pacientes con enfermedad macroquística, lo que nos alerta acerca de las indicaciones de la mastectomía subcutánea en relación a esta patología.

\section{Caso clínico}

Mujer de 26 años de edad, sin antecedentes familiares de cáncer mamario y con antecedentes personales de resección de fibroadenoma en mama derecha 4 meses antes de acudir a nuestro Servicio. Con éste único antecedente, se le propuso mastectomía subcutánea bilateral como "tratamiento preventivo" de un posible cáncer. La paciente no había sido suficientemente informada sobre las repercusiones de este procedimiento. No se le realizaron estudios de ecografía o mamografía complementarios, ni estudios genéticos de BRCA 1 ó 2.

A los 2 meses de la extirpación del fibroadenoma se le practicó mastectomía subcutánea bilateral en un

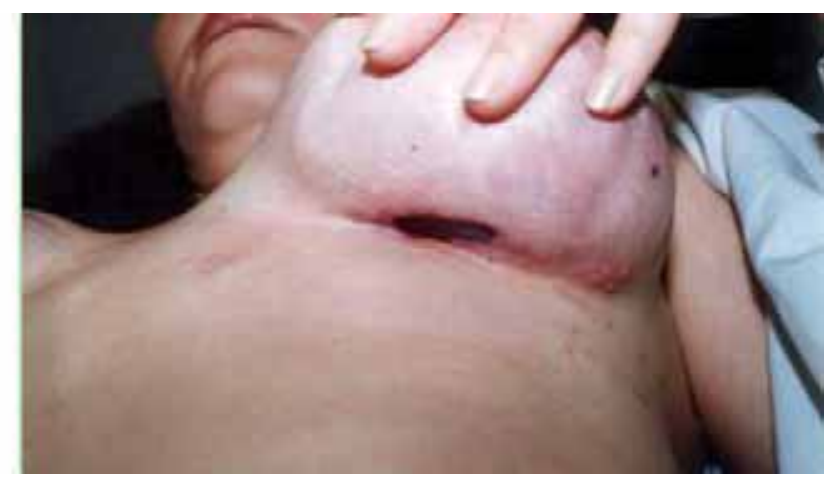

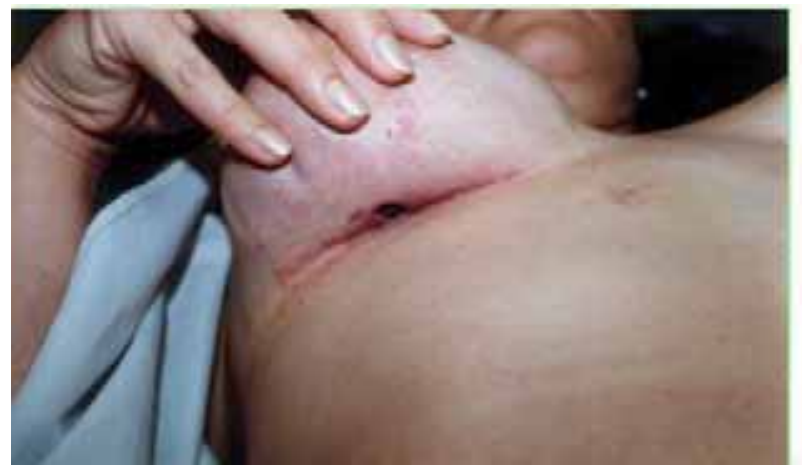

Fig. 2. Vistas basales: exposición de implantes a través de heridas dehiscentes en ambos surcos submamario. 

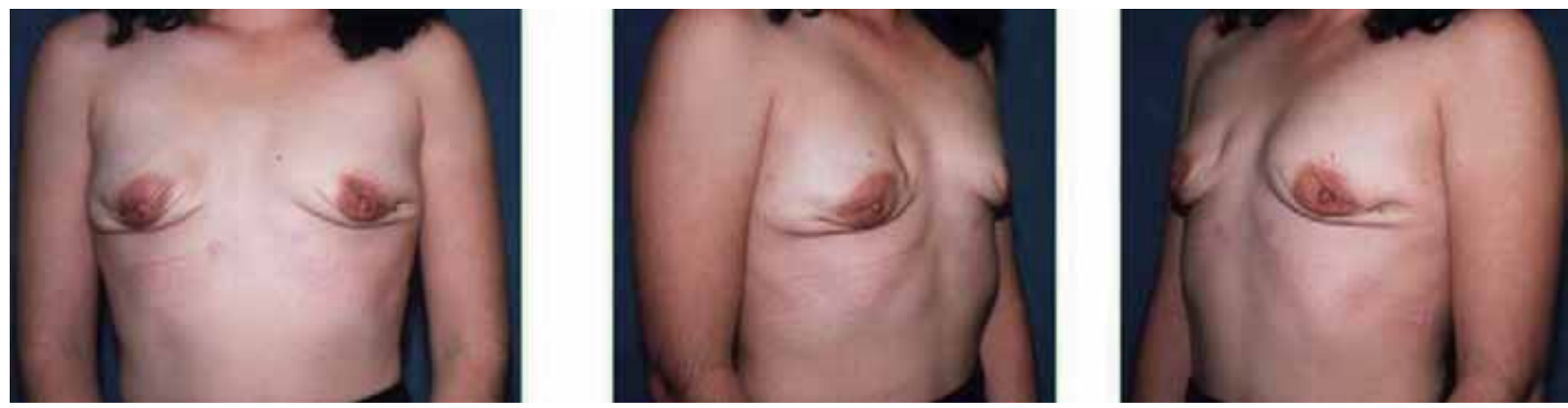

Fig. 3. Vistas frontal y de tres cuartos tras explantación de las prótesis.
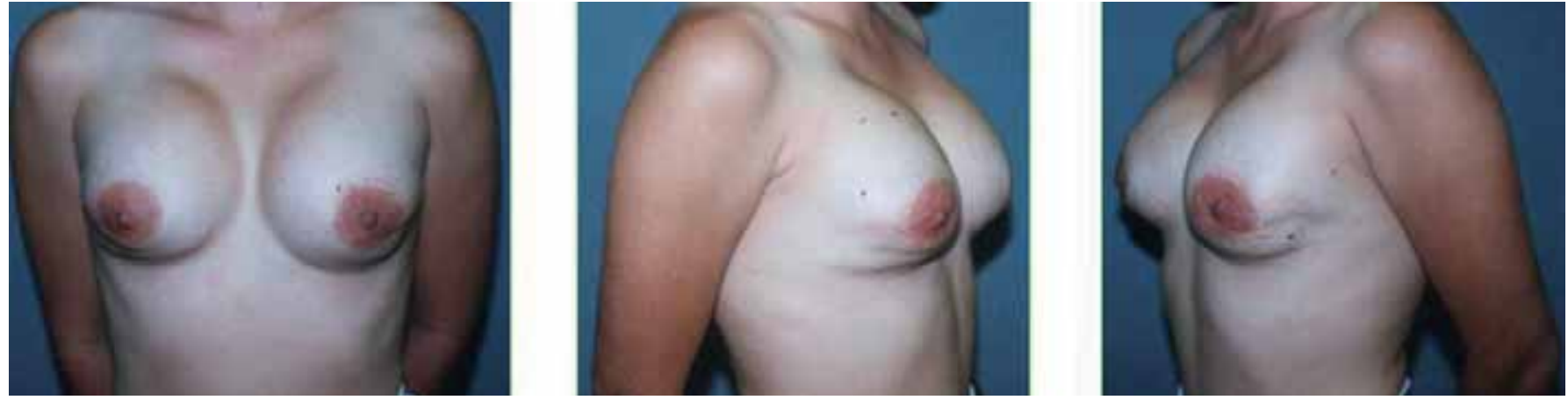

Fig. 4. Vistas frontal y de tres cuartos tras colocación de expansores mamarios retromusculares y expansión final de 320 cc.

hospital privado, por un médico oncólogo en colaboración con un cirujano plástico. En la misma intervención se le colocaron implantes mamarios de solución salina de $400 \mathrm{cc}$, redondos, texturizados, en plano subcutáneo. Cuando se le retiraron los puntos, presentó dehiscencia de herida en ambas mamas, practicándosele varios cierres secundarios sin resultado, padeciendo también eritema, aumento de la temperatura en ambas mamas y salida de material blanquecino por las heridas. En esa situación acude a nuestro Servicio; habían pasado 2 meses desde la mastectomía.

La paciente refería que no había solicitado unos implantes "tan grandes", ya que su volumen mamario tras la intervención era casi una copa más grande que el que tenía previamente (Fig. 1,2).

Se practicó tratamiento antibiótico por via oral durante 48 horas y al tercer día, lavado quirúrgico y retirada de los implantes con regularización del borde de las heridas, sutura y colocación de drenajes en circuito cerrado que se mantuvieron durante 3 días (Fig. 3). Tres meses después se colocaron expansores mamarios redondos, texturizados, de $300 \mathrm{cc}$, con válvula integradas, en plano retromuscular. Se logró una expansión final de 320 cc y se mantuvieron durante 18 meses; aunque la sustitución de los expansores por prótesis estaba prevista a los 6 meses, la paciente rechazaba nuevas cirugías (Fig. 4). En este tiempo, comenzó a presentar dolor punzante en ambas mamas, por lo que finalmente accedió a la retirada de los expansores y a la colocación de implantes de gel de silicona de $295 \mathrm{cc}$, redondos y texturizados, mediante abordaje periareolar; se practicó también en este momento, liberación de los polos mamarios inferiores, disminución del tamaño del complejo pezón-areola y capsulotomía radial bilateral.
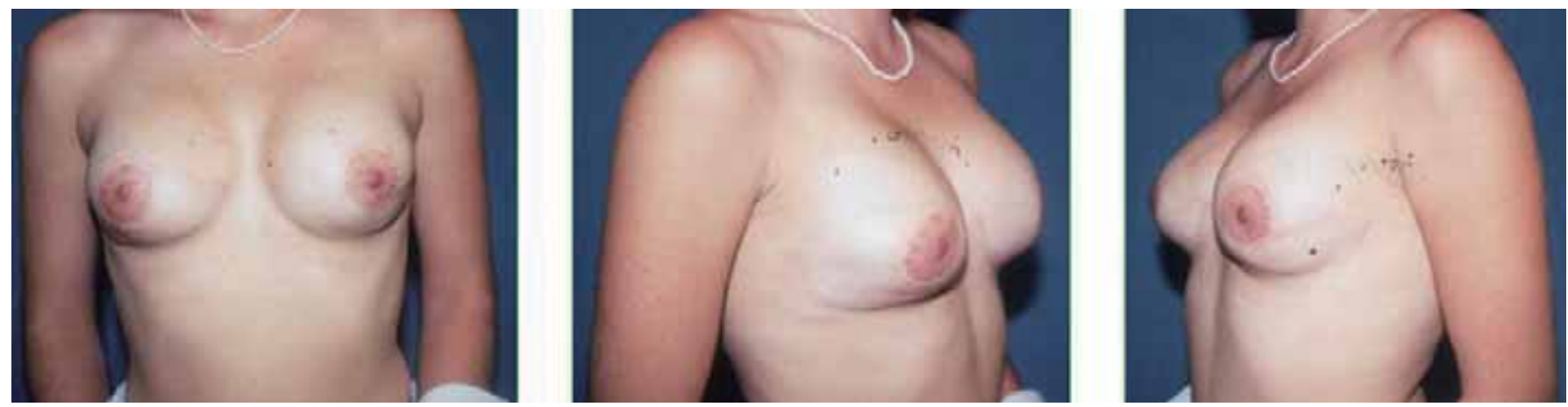

Fig. 5. Vistas frontal y de tres cuartos a los 18 meses, tras la retirada de los expansores y la colocación de prótesis mamarias de gel de silicona, redondas, texturizadas, de $295 \mathrm{cc}$. 

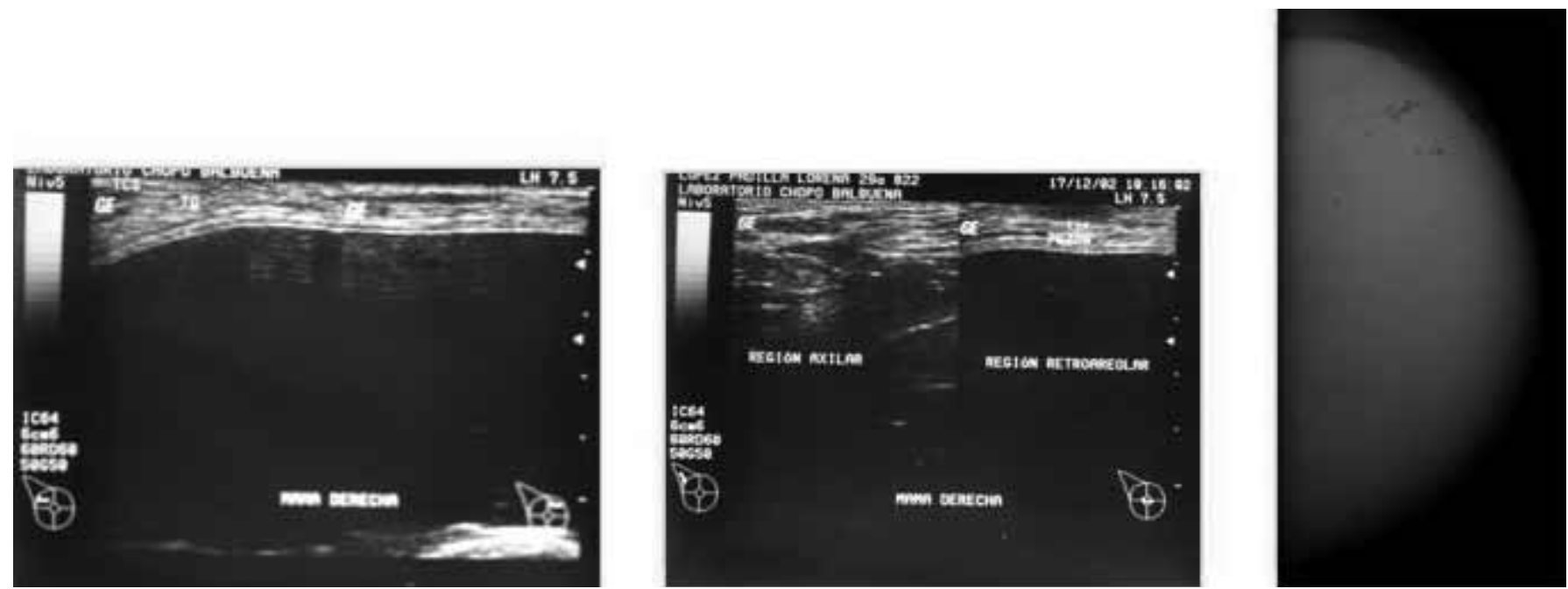

Fig. 6. Ecografía mamaria y mamografía a los 18 meses de la colocación de las prótesis. No hay evidencia de contractura capsular o ruptura de los implantes.

En el postoperatorio, se administró tratamiento antibiótico y antinflamatorio; al mes de la cirugía, se inició masaje manual y aplicación de ultrasonidos que se mantuvieron durante el $2^{\circ}$ y el $3^{\text {er }}$ mes, seguidos de Endermología durante el $4^{\circ}$ mes.

Dado que la paciente refería dolor punzante persistente, se añadió tratamiento con Colchicina durante 5 meses a dosis de $1 \mathrm{mg}$ /día, que fue rebajada a 0,5 $\mathrm{mg} / \mathrm{día}$ por intolerancia intestinal. Los 4 meses siguientes, fue tratada con Talidomida a dosis de 50 $\mathrm{mg} / \mathrm{día}$.

Dieciocho meses después de la colocación de los implantes, la paciente seguía refiriendo molestias dolorosas, sin embargo, clínicamente las mamas estaban blandas y en el estudio con ultrasonidos no aparecieron datos sugerentes de ruptura protésica ni de presencia de contractura capsular (Fig. 5, 6).

A los 3 años, la paciente presentaba contractura capsular franca y dolor importante, pero no aceptó un nuevo tratamiento quirúrgico por cambio de residencia al interior del país (Fig. 7,8). Un año más tarde, regresó solicitando tratamiento quirúrgico, momento en el que procedimos a retirar los implantes y a realizar reconstrucción mamaria con TRAM pediculado bilateral. Se le había propuesto tratamiento microqui- rúrgico para disminuir la morbilidad de la pared abdominal preservando mayor cantidad de músculo recto abdominal, pero la paciente lo rechazó. En la reconstrucción practicada no se emplearon implantes y fue necesario colocar una malla de Marlex ${ }^{\circledR}$ para cubrir el defecto de la pared anterior del abdomen una vez levantados los dos colgajos pediculados.

Presentamos imágenes del resultado postoperatorio a los 2,5 años de la reconstrucción, 8 años después de la mastectomía inicial (Fig. 9).

\section{Discusión}

Con el advenimiento de los estudios genéticos de escrutinio y el desarrollo de modelos estadísticos, hoy en día es posible identificar a las pacientes que portan un elevado riesgo de padecer cáncer mamario. Esto además abre un abanico de posibilidades de tratamiento para reducir dicho riesgo, que incluyen controles clínicos y radiológicos frecuentes, quimioprevención, salpingooforectomía profiláctica y mastectomía profiláctica. Las pacientes más beneficiadas con la mastectomía profiláctica son las portadoras del gen BRCA y aquellas con una fuerte historia familiar de cáncer mamario; también las pacientes con antece-
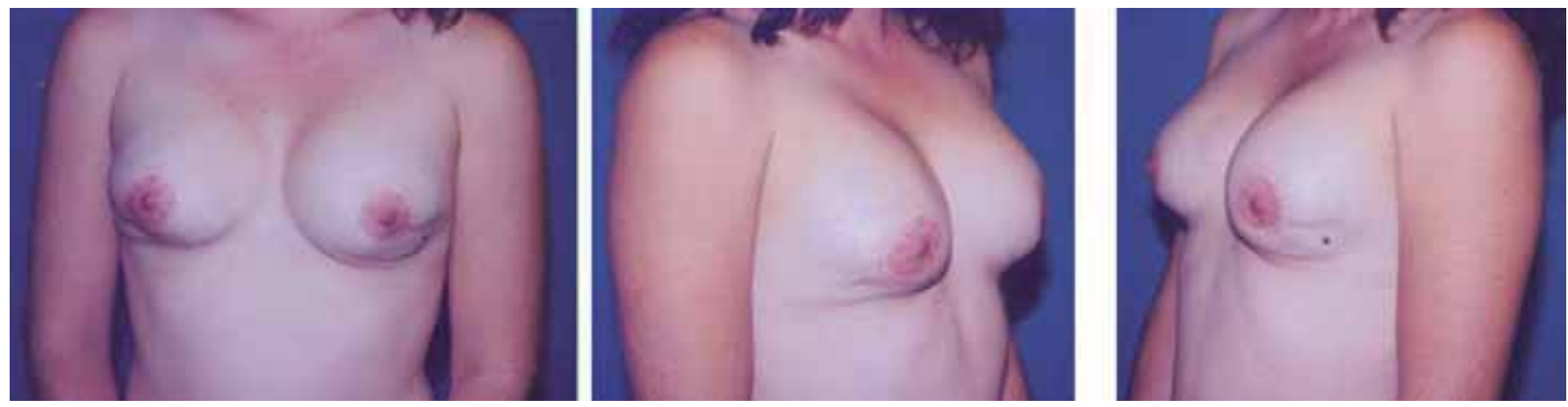

Fig. 7. Vistas frontal y de tres cuartos a los 36 meses de la colocación de las prótesis. Contractura capsular Baker grado IV. 

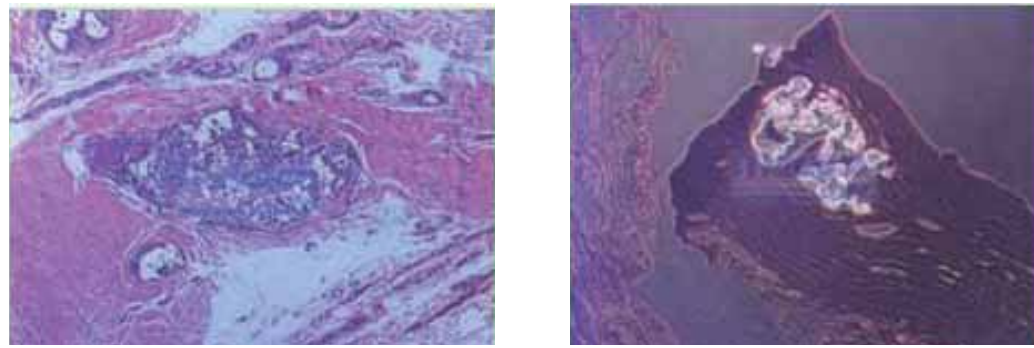

Fig. 8a: Estudio histopatológico de cápsulas mamarias derecha e izquierda. Tinción HE (Hematoxilina y Eosina). Fibrosis hialinizada, con inflamación crónica granulomatosa con formación de células multinucleadas de tipo cuerpo extraño, así como infiltrado de histiocitos. Microscopio óptico Olympus B ${ }^{8} \times 41$ aumento $4 x$.

Fig. 8b: Estudio histopatológico de cápsulas mamarias derecha e izquierda. Fibrosis hialinizada y material amorfo, anfófilo refringente y acelular. Técnica de luz polarizada en microscopio Olympus $B \AA$ con filtro especial para luz polarizada. Aumento 10x.

dentes personales de cáncer mamario puesto que presentan un alto riesgo de desarrollar un cáncer primario en la mama contralateral. Sin embargo, éste procedimiento preventivo aún está en controversia. La mastectomía profiláctica es un procedimiento irreversible, por lo que tanto el médico como la paciente deben entender sus consecuencias, beneficios, limitaciones y procedimientos alternativos disponibles (12).

Por otro lado la posibilidad de infección en un implante mamario es hasta 10 veces más elevado en pacientes con reconstrucción y depende del grado de cicatrices previas, atrofia cutánea secundaria al cáncer mamario y/o radioterapia, ambos asociados a isquemia y cicatrización retardada (13).De Cholnoky (14) reporta un incremento en la frecuencia de infecciones después de mastectomía subcutánea cuando se practica reconstrucción inmediata con implantes respecto a cuando la reconstrucción es diferida; así mismo, se considera que a diferencia de la mamoplastía de aumento con fines estéticos, el procedimiento reconstructivo se asocia a mayor incidencia de hematomas y cicatrización retardada debido a la gran disección e isquemia cutánea secundarias $(15,16)$.

En el caso que presentamos, no existía una verdadera indicación para la mastectomía subcutánea profiláctica, ni se discutieron con la paciente las posibles complicaciones de esta intervención. Tras 8 años de seguimiento desde que se le practicó la mastectomía inicial y múltiples intervenciones, la paciente presenta todavía dolores ocasionales en ambas mamas y debilitamiento de la pared abdominal que no le permite realizar ejercicios físicos; sin embargo, está satisfecha del resultado final de la reconstrucción mamaria.

Afortunadamente este tipo de casos suponen un bajo porcentaje, pero para las pacientes afectadas, este proceso supone un gran desgaste físico, económico y emocional. La indicación de una mastectomía subcutánea debe tener una plena justificación y estar documentada clínicamente; debe darse todo tipo de información a la paciente sobre los riesgos y posibles complicaciones, siempre con la valoración conjunta de un oncólogo y de un cirujano plástico para brindar la mejor alternativa posible en cada caso.

\section{Conclusiones}

La mastectomía profiláctica es un procedimiento irreversible que puede tener implicaciones severas para las pacientes, por lo que debe ser un procedimiento preventivo solo para aquellas pacientes que se encuentren dentro del grupo considerado como de alto riesgo para padecer cáncer mamario, al ser un procedimiento que no está exento de complicaciones y riesgos.

Las pacientes deben contar con una historia clínica completa, con especial énfasis en los antecedentes familiares de carcinoma mamario y estudios que respalden la indicación (ecografía, mamografía, punción biopsia con aguja fina y estudios genéticos personalizados).

La decisión de someterse a una mastectomía subcutánea profiláctica debe ser tomada de forma conjunta entre la paciente, el oncólogo y el cirujano plástico y estar plenamente justificada.
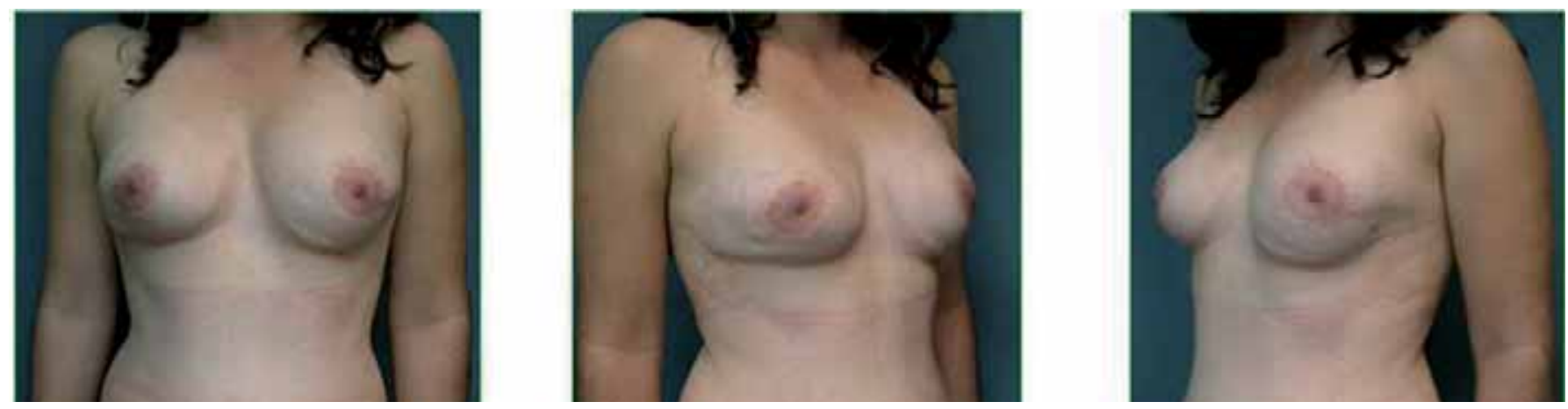

Fig. 9. Vistas frontal y de tres cuartos, 2 años y medio después de la retirada de los implantes, reconstrucción con colgajo TRAM bilateral pediculado y colocación de malla de Marlex $₫, 8$ años después de la mastectomía inicial. 


\section{Dirección del autor}

\author{
Dra. Claudia Gutiérrez Gómez \\ Hospital Médica Sur \\ Puente de Piedra \# 150 T 2 C420 \\ Col. Toriello Guerra \\ CP 14050 Tlalpan México D.F. México. \\ e-mail: clauggdelh@yahoo.com.mx
}

\section{Bibliografía}

1. Chun J et al.: "Cohort study of women at risk for breast cancer and gross cystic disease". Am J Surg 2005;190:583.

2. Metcalfe K A, Semple JL, Narod SA.: "Time to reconsider subcutaneous mastectomy for breast-cancer prevention ?". Lancet Oncol 2005;6:431.

3. Fentiman IS.: "Prophylactic mastectomy: deliverance or delusion? We don't know, so we need to start registering all cases now". BMJ 1998;317:1402.

4. Colins FS.: "BRCA1:lots of mutations, lots of dilemmas". N Engl J Med 1996;334:186.

5. Hartmann LC, Schaid DJ, Woods JE et al.: "Efficacy of bilateral prophylactic mastectomy in women with a family history of breast cancer". New Engl J Med 1999;340:77.

6. Hartmann LC, Sellers TA, Schaid DJ, et al.: "Efficacy of bilateral prophylactic mastectomy in BRCA1 and BRCA2 gene mutation carriers". J Natl Cancer Inst 2001;93:1633.
7. Meijers-Heijboer M, Van Geel B,VanPutten W, et al.: "Breast cancer after prophylactic bilateral mastectomy in women with BRCA1 or BRCA2 mutation". N Engl J Med 2001;345:158.

8. Dupont WD, Page DL.: "Risk factors for breast cancer in women with proliferative breast disease". N Eng J Med $1985 ; 312: 146$.

9. Ciatto $\mathbf{S}$ Biggeri A, Rosselli Del Turco $M$ et al.: "Risk of breast cancer subsequent to proven gross cystic disease". Eur J Cancer 1990;26:555.

10. Bundred NJ, West RR, Dowd JO, et al.: "Is there an increased risk of breast cancer in women who have had a breast cyst aspirated?" Br J Cancer 1991;64:953.

11. Dixon JM, McDonald C, Elton RA, et al.: "Risk of breast cancer in women with palpable breast cysts:a prospective study". Edinburgh Breast Group. Lancet 1999;353:1742.

12. Zakaria SH, Degnim A C: "Prophylactic Mastectomy". Surg Clin N Am 2007;87:317.

13. Pittet B, Montandon D and Pittet D.: "Infection in breast implants". Lancet Infect Dis 2005;5:94.

14. De Cholnoky T.: "Augmentation mammaplasty. Survey of complications in 10941 patients by 265 surgeons". Plast Reconstr Surg $1970 ; 45,573$.

15. Worton E, Siefert L.: "Augmentation mammaplasty: a review of fifty consecutive patients". In: Owsley JQ, Peterson RA eds. Symposium on aesthetic surgery of the breast. St Louis: Mosby.1978, Pp: 341-43.

16. Gabriel SE, Woods JE, O'Fallon WM, Beard CM, Kurland LT, Melton LJ 3rd.: "Complications leading to surgery after breast implantation”. N Eng J Med 1997;336:677. 